\title{
Simultaneous Two-Vessel Subacute Stent Thrombosis Caused by Clopidogrel Resistance from CYP2C19 Polymorphism
}

\author{
Ashwad Afzal, ${ }^{1}$ Bimal Patel, ${ }^{1}$ Neel Patel, ${ }^{2}$ Sudhakar Sattur, ${ }^{3}$ and Vinod Patel ${ }^{1}$ \\ ${ }^{1}$ Department of Internal Medicine and Division of Cardiology, New York Methodist Hospital, Brooklyn, \\ NY 11215, USA \\ ${ }^{2}$ Surat Municipal Institute of Medical Education and Research, Umarwada, Surat, Gujarat 395010, India \\ ${ }^{3}$ Department of Cardiology, Guthrie Clinic, Sayre, PA 18840, USA \\ Correspondence should be addressed to Ashwad Afzal; ashwad.afzal@gmail.com
}

Received 16 April 2016; Accepted 5 July 2016

Academic Editor: Michael S. Firstenberg

Copyright (C) 2016 Ashwad Afzal et al. This is an open access article distributed under the Creative Commons Attribution License, which permits unrestricted use, distribution, and reproduction in any medium, provided the original work is properly cited.

Clopidogrel resistance from CYP2C19 polymorphism has been associated with stent thrombosis in patients undergoing percutaneous coronary intervention with drug-eluting stents. We present a case of a 76-year-old male who received drug-eluting stents to the right coronary artery and left anterior descending artery for non-ST elevation myocardial infarction and was discharged on dual antiplatelet therapy with aspirin and clopidogrel. He subsequently presented with chest pain from anterior, anteroseptal, and inferior ST segment elevation myocardial infarction. An emergent coronary angiogram revealed acute stent thrombosis with $100 \%$ occlusion of RCA and LAD that was successfully treated with thrombus aspiration and angioplasty. Although he was compliant with his dual antiplatelet therapy, he developed stent thrombosis, which was confirmed as clopidogrel resistance from homozygous CYP2C19 polymorphism.

\section{Introduction}

Clopidogrel is recommended as part of a dual antiplatelet strategy in patients with Acute Coronary Syndrome (ACS) who receive percutaneous coronary intervention (PCI) with drug-eluting stent (DES) as it has been shown to decrease the incidence of stent thrombosis $[1,2]$. Clopidogrel is a prodrug that requires biotransformation by several hepatic cytochrome P-450 (CYP) isoenzymes including CYP2C19 to become an active metabolite [3]. However, genetic polymorphism of CYP2C19 has been associated with poor clopidogrel metabolism, in turn leading to stent thrombosis and other major adverse cardiovascular events [4].

We present a unique case report of two-vessel subacute stent thrombosis in a patient receiving clopidogrel as part of dual antiplatelet therapy after PCI with DES. This was likely due to clopidogrel resistance despite being compliant with dual antiplatelet therapy. In this report we elaborate on the details of the case and discuss diagnosis and management of patients with clopidogrel resistance.

\section{Case Presentation}

A 76-year-old male with past medical history of hypertension, hyperlipidemia, and diabetes mellitus type 2 was admitted one week priorly with chest pain due to non-ST segment elevation myocardial infarction (NSTEMI). An urgent coronary angiography showed $99 \%$ stenosis of the proximal left anterior descending artery (LAD) and 95\% stenosis of middle right coronary artery (RCA). The patient underwent PCI with DES. A $2.5 \mathrm{~mm} \times 38 \mathrm{~mm}$ Promus DES was placed in the proximal LAD. Three Promus DES were placed in the mid RCA: $2.5 \mathrm{~mm} \times 20 \mathrm{~mm}, 2.5 \mathrm{~mm} \times 28 \mathrm{~mm}$, and $2.5 \mathrm{~mm} \times$ $24 \mathrm{~mm}$ stent. His peak CK-MB and troponin were 77.4 and $107 \mathrm{ng} / \mathrm{L}$, respectively. The patient was discharged on optimal medical therapy including dual antiplatelet therapy of aspirin and clopidogrel. Due to financial constraints, ticagrelor or prasugrel could not be prescribed. He was seen one week after discharge with no complaints and was compliant with his medications.

The patient presented eight days after the index hospitalization with severe, crushing, substernal chest pressure. 


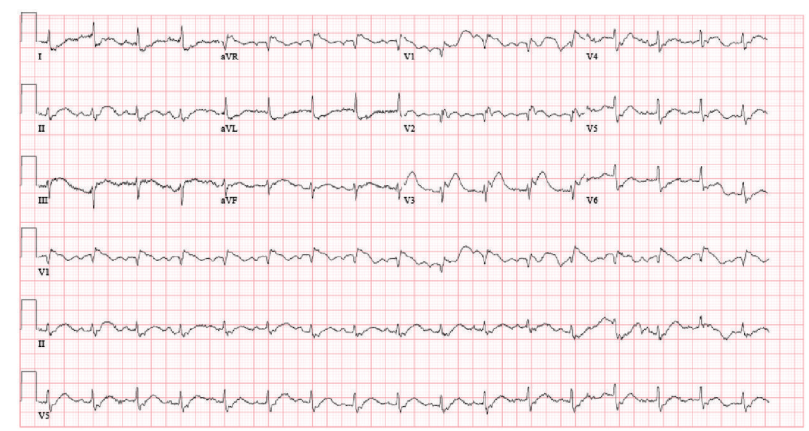

FIGURE 1: Electrocardiogram on admission showing ST segment elevation in inferior and anteroseptal distribution (STEMI from in-stent thrombosis).

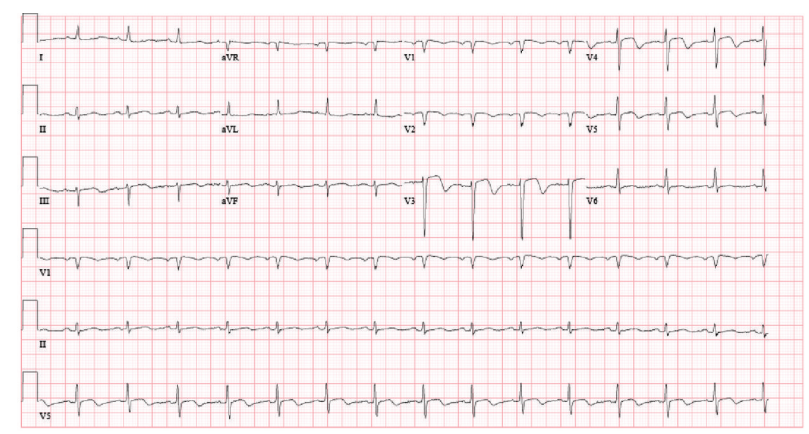

FIGURE 2: Electrocardiogram after thrombus aspiration and balloon angioplasty showing improvement of ST segment elevation in inferior and anteroseptal leads and ST segment depression in lateral leads.

The electrocardiogram showed ST segment elevation in the inferior and anteroseptal leads (Figure 1). An emergent coronary angiogram was performed revealing $100 \%$ occlusion of the proximal LAD and mid RCA due to in-stent thrombosis (Figures 3(a) and 3(b)). The patient also developed sustained ventricular tachycardia prior to PCI that was terminated with external defibrillation. Successful PCI with aspiration thrombectomy and balloon angioplasty was performed using a $2.5 \mathrm{~mm} \times 15 \mathrm{~mm}$ NC quantum Apex balloon to the proximal LAD and middle RCA lesion (Figures 4(a) and 4(b)). The patient also developed multiple episodes of symptomatic sinus bradycardia that resolved with atropine and placement of a temporary transvenous pacemaker. We suspected clopidogrel resistance; hence the patient was treated with aspirin and ticagrelor therapy. Further laboratory testing confirmed that patient had homozygous polymorphism of CYP $2 \mathrm{C} 19^{*} 2$. This is an established cause of poor clopidogrel metabolism leading to reduced levels of active clopidogrel metabolite. This was the likely cause of two-vessel stent thrombosis leading to STEMI. The patient was monitored in the cardiac intensive care unit with no further events and there was improvement in ST segment elevation on repeat electrocardiogram (Figure 2). A transthoracic echocardiogram showed severe diffuse hypokinesis with abnormal left ventricular relaxation, an ejection fraction of $20 \%$, and no significant valvulopathies. His peak CK-MB and troponin were 113 and $23.9 \mathrm{ng} / \mathrm{L}$, respectively. He was recovering well without any further cardiac events at one-month and three-month clinic follow-up.

\section{Discussion}

Clopidogrel is a prodrug that requires activation by the hepatic cytochrome P-450 system to become an active metabolite [3]. This active metabolite inhibits platelet aggregation by irreversibly binding to platelet ADP receptor P2Y12. Genetic polymorphism of the cytochrome P-450 isoenzymes can affect clopidogrel metabolism. Polymorphism with loss-offunction alleles CYP2C19*2 (681G $>$ A) is associated with poor clopidogrel metabolism that is seen in both heterozygous and homozygous patients [5-7]. This defect leads to a mutation in exon 5 that creates an aberrant splice site resulting in a truncated, nonfunctional protein [8]. Mega et al. showed that carriers of at least one CYP2C19 reducedfunction allele had a $32.4 \%$ relative reduction in active clopidogrel metabolite in comparison to noncarriers $(P<$ 0.001) [4]. A low circulating active clopidogrel metabolite causes a diminished platelet response to treatment leading to higher rates of major adverse cardiovascular events [4, 9]. Genotype analysis in this patient showed homozygous lossof-function alleles of CYP $2 \mathrm{C} 9^{*} 2 /{ }^{*} 2$. This led to inadequate platelet inhibition resulting in two-vessel stent thrombosis. In patients with heterozygous CYP2C19*2 alleles, higher doses of clopidogrel have been shown to overcome the genetic 


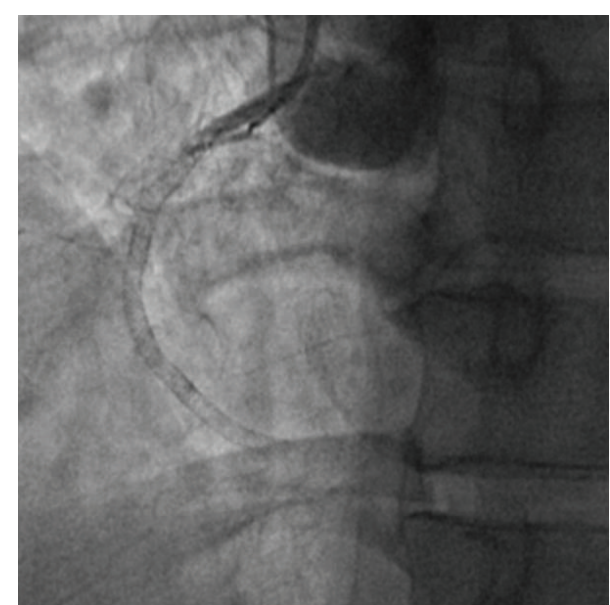

(a) RCA (LAO view)

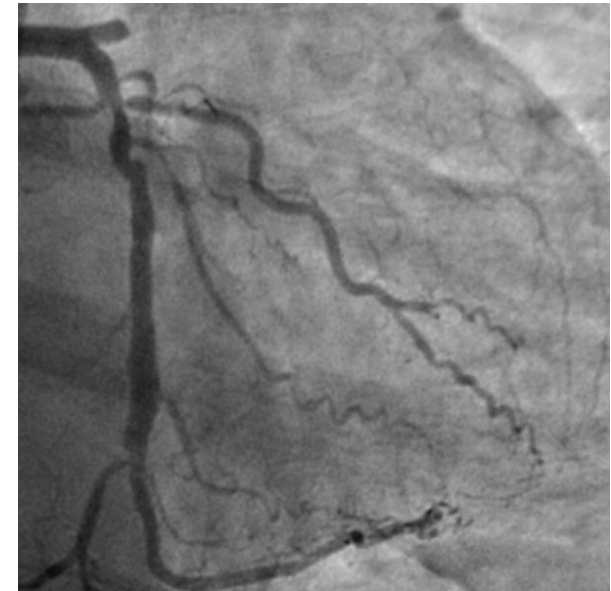

(b) LAD (RAO caudal view)

FIgURE 3: Angiogram revealing in-stent thrombosis of the RCA and LAD.

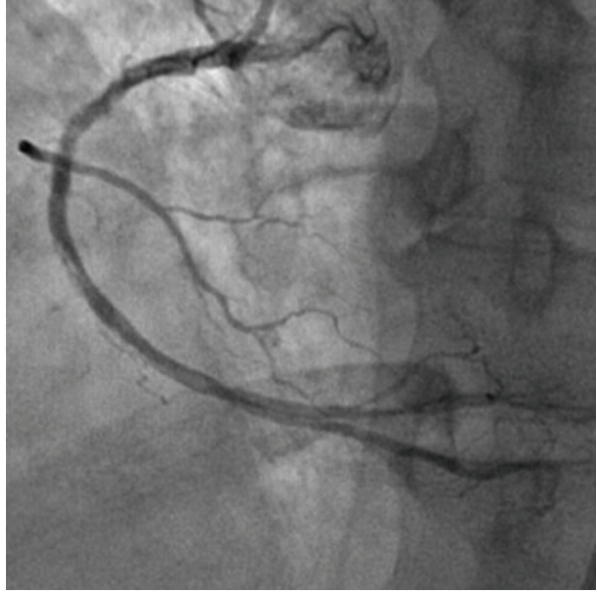

(a) RCA (LAO view)

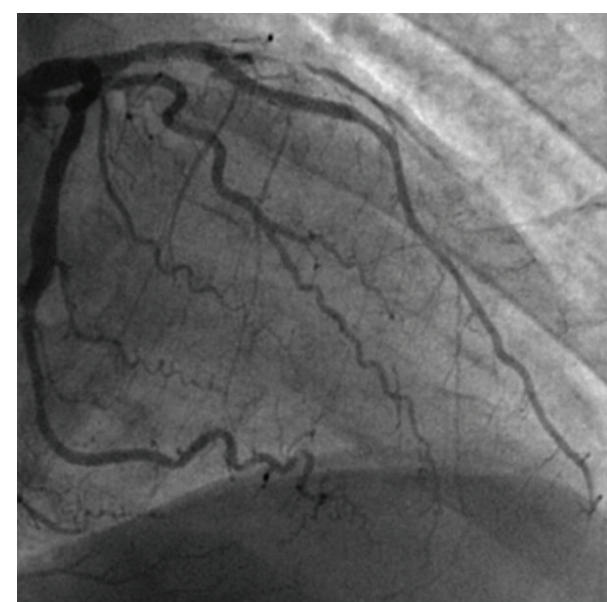

(b) LAD (RAO cranial view)

FIGURE 4: Angiogram after thrombus aspiration and balloon angioplasty of the RCA and LAD.

resistance and improve the platelet inhibition [10]. However, higher doses of clopidogrel cannot overcome the resistance and are not an option for CYP2C19*2 homozygote patients. An alternative antiplatelet therapy with ticagrelor was used. Ticagrelor is a reversible inhibitor of ADP receptor P2Y12 that has been shown to have a greater and more consistent inhibition of platelet aggregation in comparison to standard clopidogrel dose [11]. It has been shown to reduce the rate of death from myocardial infarction compared to clopidogrel without a significant increase in major bleeding [12].

\section{Competing Interests}

The authors declare that there is no conflict of interests regarding the publication of this paper.

\section{References}

[1] M. E. Bertrand, H.-J. Rupprecht, P. Urban, and A. H. Gershlick, "Double-blind study of the safety of clopidogrel with and without a loading dose in combination with aspirin compared with ticlopidine in combination with aspirin after coronary stenting: the clopidogrel aspirin stent international cooperative study (CLASSICS)," Circulation, vol. 102, no. 6, pp. 624-629, 2000.

[2] S. R. Mehta, S. Yusuf, R. J. G. Peters et al., "Effects of pretreatment with clopidogrel and aspirin followed by long-term therapy in patients undergoing percutaneous coronary intervention: the PCI-CURE study," The Lancet, vol. 358, no. 9281, pp. 527-533, 2001.

[3] P. Savi, J. M. Herbert, A. M. Pflieger et al., "Importance of hepatic metabolism in the antiaggregating activity of the thienopyridine clopidogrel," Biochemical Pharmacology, vol. 44, no. 3, pp. 527-532, 1992.

[4] J. L. Mega, S. L. Close, S. D. Wiviott et al., "Cytochrome P-450 polymorphisms and response to clopidogrel," The New England Journal of Medicine, vol. 360, no. 4, pp. 354-362, 2009.

[5] J.-S. Hulot, A. Bura, E. Villard et al., "Cytochrome P450 2C19 loss-of-function polymorphism is a major determinant of clopidogrel responsiveness in healthy subjects," Blood, vol. 108, no. 7, pp. 2244-2247, 2006. 
[6] K. Umemura, T. Furuta, and K. Kondo, "The common gene variants of CYP2C19 affect pharmacokinetics and pharmacodynamics in an active metabolite of clopidogrel in healthy subjects," Journal of Thrombosis and Haemostasis, vol. 6, no. 8, pp. 1439-1441, 2008.

[7] Z. Desta, X. Zhao, J.-G. Shin, and D. A. Flockhart, "Clinical significance of the cytochrome P450 2C19 genetic polymorphism," Clinical Pharmacokinetics, vol. 41, no. 12, pp. 913-958, 2002.

[8] S. M. F. de Morais, G. R. Wilkinson, J. Blaisdell, K. Nakamura, U. A. Meyer, and J. A. Goldstein, "The major genetic defect responsible for the polymorphism of S-mephenytoin metabolism in humans," The Journal of Biological Chemistry, vol. 269, no. 22, pp. 15419-15422, 1994.

[9] J. T. Brandt, S. L. Close, S. J. Iturria et al., "Common polymorphisms of CYP2C19 and CYP2C9 affect the pharmacokinetic and pharmacodynamic response to clopidogrel but not prasugrel," Journal of Thrombosis and Haemostasis, vol. 5, no. 12, pp. 2429-2436, 2007.

[10] J.-P. Collet, J.-S. Hulot, G. Anzaha et al., "High doses of clopidogrel to overcome genetic resistance: the randomized crossover CLOVIS-2 (Clopidogrel and Response Variability Investigation Study 2)," JACC: Cardiovascular Interventions, vol. 4, no. 4, pp. 392-402, 2011.

[11] R. F. Storey, S. Husted, R. A. Harrington et al., "Inhibition of platelet aggregation by AZD6140, a reversible oral P2Y12 receptor antagonist, compared with clopidogrel in patients with acute coronary syndromes," Journal of the American College of Cardiology, vol. 50, no. 19, pp. 1852-1856, 2007.

[12] L. Wallentin, R. C. Becker, A. Budaj et al., "Ticagrelor versus clopidogrel in patients with acute coronary syndromes," The New England Journal of Medicine, vol. 361, no. 11, pp. 1045-1057, 2009. 


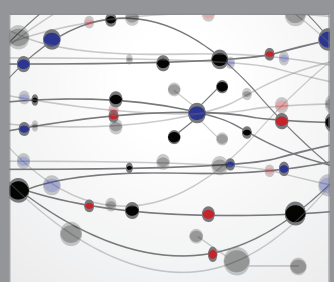

The Scientific World Journal
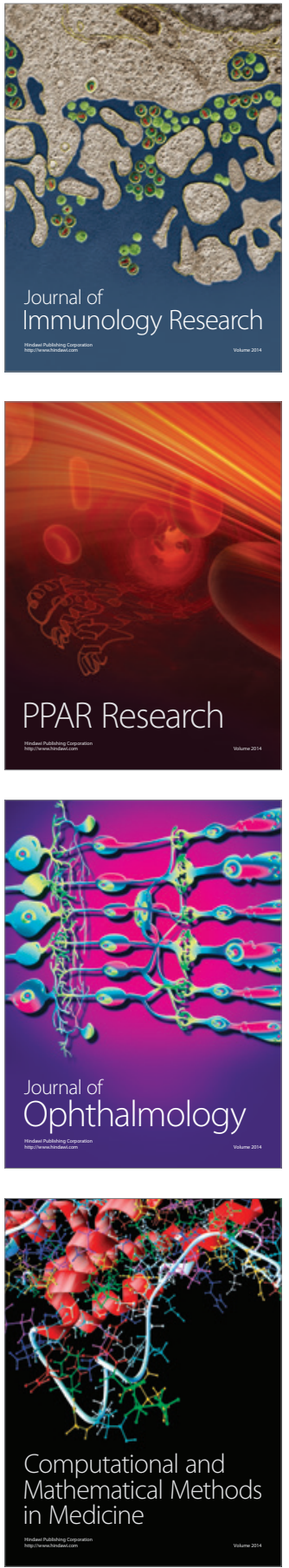

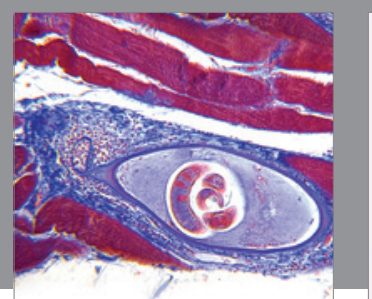

Gastroenterology Research and Practice

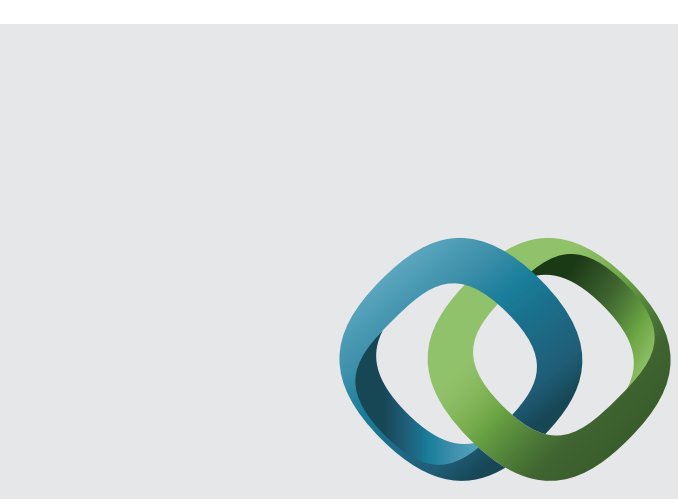

\section{Hindawi}

Submit your manuscripts at

http://www.hindawi.com
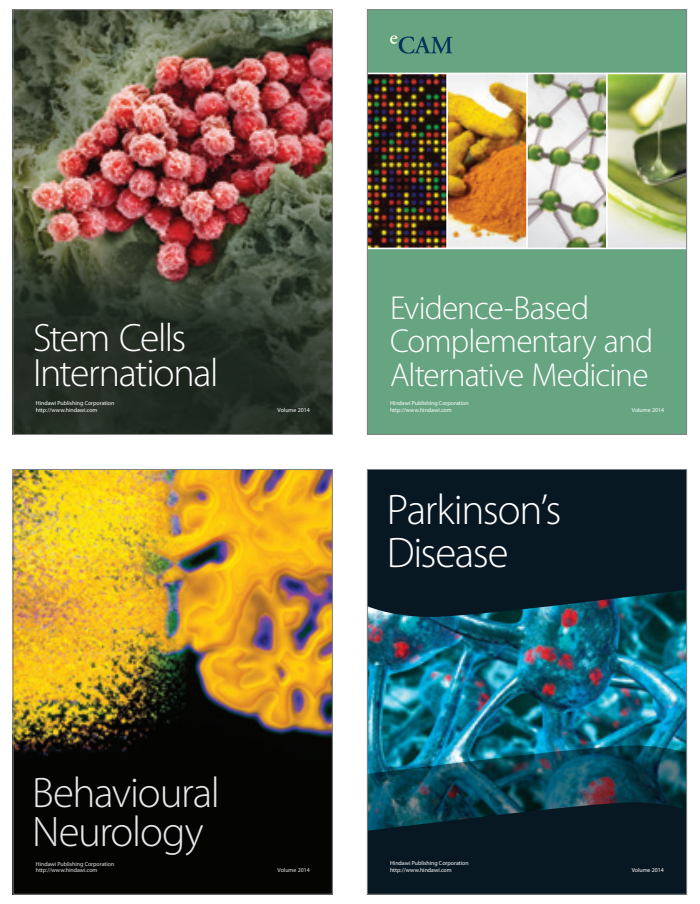
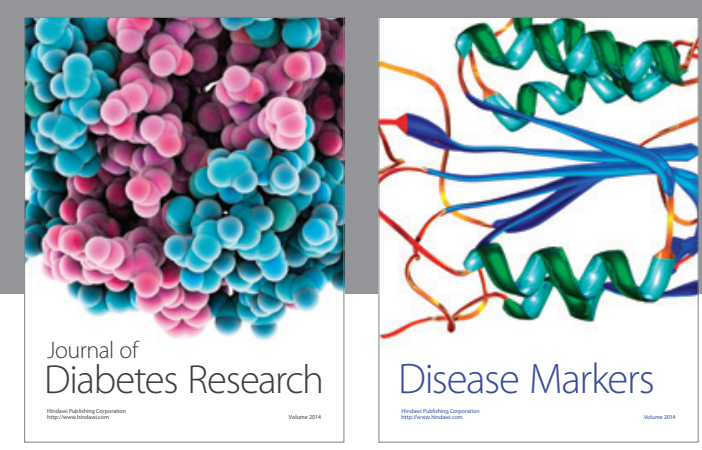

Disease Markers
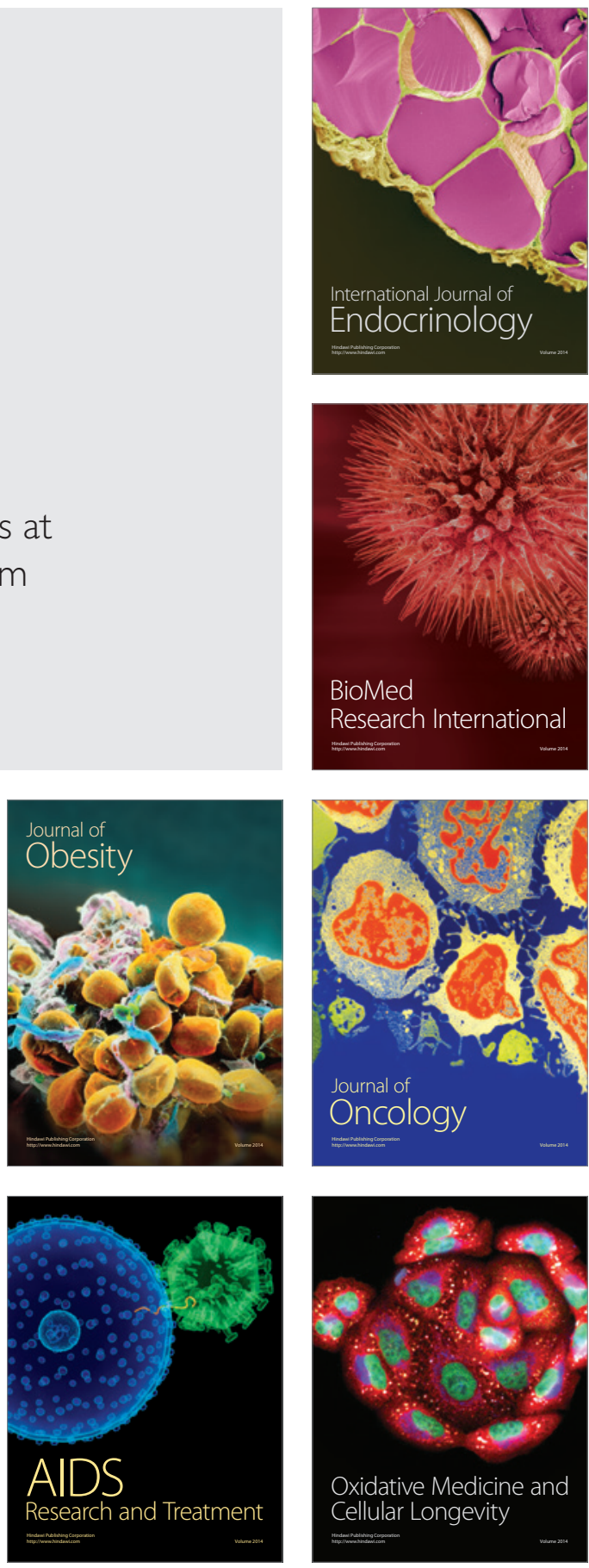